doi:10.13108/2016-8-2-95

\title{
SOME RESULTS ON GENERALIZED RELATIVE ORDERS OF MEROMORPHIC FUNCTIONS
}

\author{
S.K. DATTA, T. BISWAS, P. DAS
}

\begin{abstract}
In this paper we discuss some growth rates of compositions of entire and meromorphic functions on the base of generalized relative order and generalized relative lower order of meromorphic functions with respect to entire functions.
\end{abstract}

Keywords: meromorphic function, entire function, generalized relative order, generalized relative lower order, composition, growth.

Mathematics Subject Classification: 30D35, 30D30, 30D20

\section{Introduction, Definitions and Notations}

Let $f$ be an entire function defined in the complex plane $\mathbb{C}$. The maximum modulus function corresponding to entire function $f$ is defined as $M_{f}(r)=\max \{|f(z)|:|z|=r\}$. If $f$ is nonconstant, it has the following property:

Property (A) [2] : A non-constant entire function $f$ is said to have Property (A) if for any $\sigma>1$ and for all sufficiently large values of $r,\left[M_{f}(r)\right]^{2} \leqslant M_{f}\left(r^{\sigma}\right)$ holds. For examples of functions with or without the Property (A), one may see [2].

When $f$ is meromorphic, $M_{f}(r)$ can not be defined as $f$ is not analytic. In this case one may define another function $T_{f}(r)$ known as Nevanlinna's Characteristic function of $f$, playing the same role as maximum modulus function, in the following manner:

$$
T_{f}(r)=N_{f}(r)+m_{f}(r),
$$

where functions $N_{f}(r)$ and $m_{f}(r)$ are defined as follows. We first introduce function $N_{f}(r, a)\left(\bar{N}_{f}(r, a)\right)$ known as the counting function of a-points (distinct $a$-points) of meromorphic $f$ as

$$
\begin{aligned}
& N_{f}(r, a)=\int_{0}^{r} \frac{n_{f}(t, a)-n_{f}(0, a)}{t} d t+\bar{n}_{f}(0, a) \log r \\
& \left(\bar{N}_{f}(r, a)=\int_{0}^{r} \frac{\bar{n}_{f}(t, a)-\bar{n}_{f}(0, a)}{t} d t+\bar{n}_{f}(0, a) \log r\right),
\end{aligned}
$$

moreover, we denote by $n_{f}(r, a)\left(\bar{n}_{f}(r, a)\right)$ the number of $a$-points (distinct $a$-points) of $f$ in $|z| \leqslant r$ and an $\infty$-point is a pole of $f$. In many situations, $N_{f}(r, \infty)$ and $\bar{N}_{f}(r, \infty)$ are denoted by $N_{f}(r)$ and $\bar{N}_{f}(r)$, respectively.

S.K. Datta, T. Biswas, P. Das, Some results focusing generalized Relative orders of MEROMORPHIC FUNCTIONS.

(c) S.K. Datta, T. Biswas, P. Das 2016.

Поступила 22 июля 2015 г. 
Function $m_{f}(r, \infty)$ alternatively denoted by $m_{f}(r)$ known as the proximity function of $f$ is defined as

$$
\begin{aligned}
& m_{f}(r)=\frac{1}{2 \pi} \int_{0}^{2 \pi} \log ^{+}\left|f\left(r e^{i \theta}\right)\right| d \theta, \quad \text { where } \\
& \log ^{+} x=\max (\log x, 0) \text { for all } x \geqslant 0 .
\end{aligned}
$$

Also we may denote $m\left(r, \frac{1}{f-a}\right)$ by $m_{f}(r, a)$.

If $f$ is entire function, then the Nevanlinna's Characteristic function $T_{f}(r)$ of $f$ is defined as

$$
T_{f}(r)=m_{f}(r) \text {. }
$$

For any two entire functions $f$ and $g$, the ratio $\frac{M_{f}(r)}{M_{g}(r)}$ as $r \rightarrow \infty$ is called the growth of $f$ with respect to $g$ in terms of their maximum moduli. Also the ratio $\frac{T_{f}(r)}{T_{g}(r)}$ as $r \rightarrow \infty$ is called the growth of $f$ with respect to $g$ in terms of the Nevanlinna's Characteristic functions, when $f$ and $g$ are both meromorphic functions. Accordingly, the study of comparative growth properties of entire and meromorphic functions which is one of the prominent branches of the value distribution theory of entire and meromorphic functions is the prime concern of the paper. We do not explain the standard definitions and notations in the theory of entire and meromorphic functions as those are available in [12] and [15]. In the sequel the following two notations are used:

$$
\begin{aligned}
& \log ^{[k]} x=\log \left(\log ^{[k-1]} x\right) \text { for } k=1,2,3, \cdots \\
& \log ^{[0]} x=x
\end{aligned}
$$

and

$$
\begin{aligned}
& \exp ^{[k]} x=\exp \left(\exp ^{[k-1]} x\right) \text { for } k=1,2,3, \cdots ; \\
& \exp ^{[0]} x=x
\end{aligned}
$$

Taking this into account, the generalized order (respectively, generalized lower order) of an entire function $f$ as introduced by Sato [14] is given by:

$$
\begin{aligned}
& \rho_{f}^{[l]}=\limsup _{r \rightarrow \infty} \frac{\log ^{[l]} M_{f}(r)}{\log \log M_{\exp z}(r)}=\limsup _{r \rightarrow \infty} \frac{\log ^{[l]} M_{f}(r)}{\log r}, \\
& \left(\text { respectively, } \lambda_{f}^{[l]}=\liminf _{r \rightarrow \infty} \frac{\log ^{[l]} M_{f}(r)}{\log \log M_{\exp z}(r)}=\liminf _{r \rightarrow \infty} \frac{\log ^{[l]} M_{f}(r)}{\log r}\right),
\end{aligned}
$$

where $l \geqslant 1$.

When $f$ is meromorphic function, one can easily verify that

$$
\begin{aligned}
& \rho_{f}^{[l]}=\limsup _{r \rightarrow \infty} \frac{\log ^{[l-1]} T_{f}(r)}{\log T_{\exp z}(r)}=\limsup _{r \rightarrow \infty} \frac{\log ^{[l-1]} T_{f}(r)}{\log \left(\frac{r}{\pi}\right)}=\limsup _{r \rightarrow \infty} \frac{\log ^{[l-1]} T_{f}(r)}{\log r+O(1)}, \\
& \left(\text { respectively, } \lambda_{f}^{[l]}=\liminf _{r \rightarrow \infty} \frac{\log ^{[l-1]} T_{f}(r)}{\log T_{\exp z}(r)}=\liminf _{r \rightarrow \infty} \frac{\log ^{[l-1]} T_{f}(r)}{\log r+O(1)}\right),
\end{aligned}
$$

where $l \geqslant 1$.

These definitions extend the definitions of order $\rho_{f}$ and lower order $\lambda_{f}$ of an entire and meromorphic function $f$ since for $l=2$, these correspond to the particular case $\rho_{f}^{[2]}=\rho_{f}$ and $\lambda_{f}^{[2]}=\lambda_{f}$. 
Given a non-constant entire function $g$ defined in the complex plane $\mathbb{C}$, its maximum modulus function $M_{g}(r)$ and Nevanlinna's Characteristic function $T_{g}(r)$ are both strictly increasing and continuous functions of $r$. Also their inverses $M_{g}^{-1}(r):(|g(0)|, \infty) \rightarrow(0, \infty)$ and $T_{g}^{-1}$ : $\left(T_{g}(0), \infty\right) \rightarrow(0, \infty)$ exist and are such that $\lim _{s \rightarrow \infty} M_{g}^{-1}(s)=\infty$ and $\lim _{s \rightarrow \infty} T_{g}^{-1}(s)=\infty$.

Extending the idea of relative order of entire functions as established by Bernal [1], 2], Lahiri and Banerjee [13] introduced the definition of relative order of a meromorphic function $f$ with respect to another entire function $g$, denoted by $\rho_{g}(f)$ to avoid comparing growth just with $\exp z$ as follows:

$$
\begin{aligned}
\rho_{g}(f) & =\inf \left\{\mu>0: T_{f}(r)<T_{g}\left(r^{\mu}\right) \text { for all sufficiently large } r\right\} \\
& =\limsup _{r \rightarrow \infty} \frac{\log T_{g}^{-1} T_{f}(r)}{\log r} .
\end{aligned}
$$

The definition coincides with the classical one if $g(z)=\exp z$, cf. [14].

Likewise, one can define the relative lower order of a meromorphic function $f$ with respect to an entire function $g$ denoted by $\lambda_{g}(f)$ as follows :

$$
\lambda_{g}(f)=\liminf _{r \rightarrow \infty} \frac{\log T_{g}^{-1} T_{f}(r)}{\log r} .
$$

Further, Banerjee and Jana [4] gave a more generalized concept of relative order of a meromorphic function with respect to an entire function in the following way:

Definition 1.1. [4] If $l \geqslant 1$ is a positive integer, then the $l$-th generalized relative order of a meromorphic function $f$ with respect to an entire function $g$ denoted by $\rho_{g}^{[l]}(f)$ is defined by

$$
\rho_{g}^{[l]}(f)=\limsup _{r \rightarrow \infty} \frac{\log ^{[l]} T_{g}^{-1} T_{f}(r)}{\log r} .
$$

Likewise one can define the generalized relative lower order of a meromorphic function $f$ with respect to an entire function $g$ denoted by $\lambda_{g}^{[l]}(f)$ as

$$
\lambda_{g}^{[l]}(f)=\liminf _{r \rightarrow \infty} \frac{\log ^{[l]} T_{g}^{-1} T_{f}(r)}{\log r} .
$$

For entire and meromrophic functions, the notions of their growth indicators such as order is classical in complex analysis and during the past decades, several researchers have already been exploring their studies in the area of comparative growth properties of compositions of entire and meromorphic functions in different directions using the classical growth indicators. But at that time, the concepts of relative orders andconsequently, the generalized relative orders of entire and meromorphic functions with respect to another entire function and as well as their technical advantages of not comparing with the growths of exp $z$ were not at all known to the researchers of this area. Therefore the growth of compositions of entire and meromorphic functions needs to be modified on the basis of their relative order some of which has been explored in [5], 6], 7], 8], 9], [10] and [11. In this paper we establish some newly developed results related to the growth rates of composite entire and meromorphic functions on the basis of their generalized relative orders (respectively, generalized relative lower orders).

\section{LEMMA}

In this section we present a lemma which will be needed in the sequel.

Lemma 2.1. [3] Let $f$ be meromorphic and $g$ be entire and suppose that $0<\mu<\rho_{g} \leqslant \infty$. Then for a sequence of values of $r$ tending to infinity,

$$
T_{f \circ g}(r) \geqslant T_{f}\left(\exp \left(r^{\mu}\right)\right) .
$$




\section{THEOREMS}

In this section we present the main results of the paper.

Theorem 3.1. Let $f$ be a meromorphic function and $g, h$ be any two entire functions such that

and

$$
\text { (i) } \liminf _{r \rightarrow \infty} \frac{\log ^{[l]} T_{h}^{-1}(r)}{(\log r)^{\alpha}}=A, \quad \text { a is a real number }>0
$$

$$
\text { (ii) } \liminf _{r \rightarrow \infty} \frac{\log ^{[l]} T_{h}^{-1} T_{f}\left(\exp r^{\mu}\right)}{\left(\log ^{[l]} T_{h}^{-1}(r)\right)^{\beta+1}}=B, \quad \text { a is a real number }>0
$$

for any $\alpha, \beta, \mu$ satisfying $0<\alpha<1, \beta>0, \alpha(\beta+1)>1$ and $0<\mu<\rho_{g} \leqslant \infty$. Then

$$
\rho_{h}^{[l]}(f \circ g)=\infty
$$

where $l$ is any positive integer.

Proof. From $(i)$ we have for all sufficiently large values of $r$ that

$$
\log ^{[l]} T_{h}^{-1}(r) \geqslant(A-\varepsilon)(\log r)^{\alpha}
$$

and from $(i i)$ we obtain for all sufficiently large values of $r$ that

$$
\log ^{[l]} T_{h}^{-1} T_{f}\left(\exp r^{\mu}\right) \geqslant(B-\varepsilon)\left(\log ^{[l]} T_{h}^{-1}(r)\right)^{\beta+1} .
$$

Also $T_{h}^{-1}(r)$ is an increasing function of $r$ and it follows from (3.1), (3.2) and Lemma 2.1 for a sequence of values of $r$ tending to infinity that

$$
\begin{aligned}
& \log ^{[l]} T_{h}^{-1} T_{f \circ g}(r) \geqslant \log ^{[l]} T_{h}^{-1} T_{f}\left(\exp \left(r^{\mu}\right)\right), \\
& \log ^{[l]} T_{h}^{-1} T_{f \circ g}(r) \geqslant(B-\varepsilon)\left(\log ^{[l]} T_{h}^{-1}(r)\right)^{\beta+1}, \\
& \log ^{[l]} T_{h}^{-1} T_{f \circ g}(r) \geqslant(B-\varepsilon)\left[(A-\varepsilon)(\log r)^{\alpha}\right]^{\beta+1}, \\
& \log ^{[l]} T_{h}^{-1} T_{f \circ g}(r) \geqslant(B-\varepsilon)(A-\varepsilon)^{\beta+1}(\log r)^{\alpha(\beta+1)}, \\
& \frac{\log ^{[l]} T_{h}^{-1} T_{f \circ g}(r)}{\log r} \geqslant \frac{(B-\varepsilon)(A-\varepsilon)^{\beta+1}(\log r)^{\alpha(\beta+1)}}{\log r}, \\
& \limsup _{r \rightarrow \infty} \frac{\log [l] T_{h}^{-1} T_{f \circ g}(r)}{\log r} \geqslant \liminf _{r \rightarrow \infty} \frac{(B-\varepsilon)(A-\varepsilon)^{\beta+1}(\log r)^{\alpha(\beta+1)}}{\log r} .
\end{aligned}
$$

Since $\varepsilon>0$ is arbitrary and $\alpha(\beta+1)>1$, it follows from above that

$$
\rho_{h}^{[l]}(f \circ g)=\infty \text {. }
$$

This proves the theorem.

Theorem 3.2. Let $f$ be a meromorphic function and $g, h$ be any two entire functions such that

$$
\text { (i) } \liminf _{r \rightarrow \infty} \frac{\log ^{[l]} T_{h}^{-1}\left(\exp \left(r^{\mu}\right)\right)}{\left(\log ^{[2]} r\right)^{\alpha}}=A, \quad \text { a is a real number }>0
$$

and

$$
\text { (ii) } \liminf _{r \rightarrow \infty} \frac{\log \left[\frac{\log \left[{ }^{[l]} T_{h}^{-1}\left(T_{f}\left(\exp r^{\mu}\right)\right)\right.}{\log T_{h}^{l l}\left(\exp r^{\mu}\right)}\right]}{\left[\log ^{[l]} T_{h}^{-1}\left(\exp r^{\mu}\right)\right]^{\beta}}=B, \quad \text { a is a real number }>0
$$


for any $\alpha, \beta$ satisfying $\alpha>1,0<\beta<1, \alpha \beta>1$ and $0<\mu<\rho_{g} \leqslant \infty$. Then

$$
\rho_{h}^{[l]}(f \circ g)=\infty \text {, }
$$

where $l$ is any integer with $l \geqslant 1$.

Proof. From $(i)$ we have for all sufficiently large values of $r$ that

$$
\log ^{[l]} T_{h}^{-1}\left(\exp \left(r^{\mu}\right)\right) \geqslant\left((A-\varepsilon) \log ^{[2]} r\right)^{\alpha}
$$

and from $(i i)$ we obtain for all sufficiently large values of $r$ that

$$
\begin{aligned}
& \log \left[\frac{\log ^{[l]} T_{h}^{-1}\left(T_{f}\left(\exp r^{\mu}\right)\right)}{\log { }^{[l]} T_{h}^{-1}\left(\exp r^{\mu}\right)}\right] \geqslant(B-\varepsilon)\left[\log ^{[l]} T_{h}^{-1}\left(\exp r^{\mu}\right)\right]^{\beta}, \\
& \frac{\log ^{[l]} T_{h}^{-1}\left(T_{f}\left(\exp r^{\mu}\right)\right)}{\log ^{[l]} T_{h}^{-1}\left(\exp r^{\mu}\right)} \geqslant \exp \left[(B-\varepsilon)\left[\log ^{[l]} T_{h}^{-1}\left(\exp r^{\mu}\right)\right]^{\beta}\right] .
\end{aligned}
$$

Also $T_{h}^{-1}(r)$ is an increasing function of $r$ and it follows from(3.3), (3.4) and Lemma 2.1 for a sequence of values of $r$ tending to infinity that

$$
\begin{aligned}
& \frac{\log ^{[l]} T_{h}^{-1} T_{f \circ g}(r)}{\log r} \geqslant \frac{\log ^{[l]} T_{h}^{-1} T_{f}\left(\exp \left(r^{\mu}\right)\right)}{\log r}, \\
& \frac{\log ^{[l]} T_{h}^{-1} T_{f \circ g}(r)}{\log r} \geqslant \frac{\log ^{[l]} T_{h}^{-1} T_{f}\left(\exp \left(r^{\mu}\right)\right)}{\log ^{[l]} T_{h}^{-1}\left(\exp \left(r^{\mu}\right)\right)} \cdot \frac{\log ^{[l]} T_{h}^{-1}\left(\exp \left(r^{\mu}\right)\right)}{\log r}, \\
& \frac{\log ^{[l]} T_{h}^{-1} T_{f \circ g}(r)}{\log r} \geqslant \exp \left[(B-\varepsilon)\left[\log ^{[l]} T_{h}^{-1}\left(\exp r^{\mu}\right)\right]^{\beta}\right] \cdot \frac{(A-\varepsilon)\left(\log ^{[2]} r\right)^{\alpha}}{\log r}, \\
& \frac{\log ^{[l]} T_{h}^{-1} T_{f \circ g}(r)}{\log r} \geqslant \exp \left[(B-\varepsilon)(A-\varepsilon)^{\beta}\left(\log ^{[2]} r\right)^{\alpha \beta}\right] \cdot \frac{(A-\varepsilon)\left(\log ^{[2]} r\right)^{\alpha}}{\log r}, \\
& \frac{\log ^{[l]} T_{h}^{-1} T_{f \circ g}(r)}{\log r} \geqslant \exp \left[(B-\varepsilon)(A-\varepsilon)^{\beta}\left(\log ^{[2]} r\right)^{\alpha \beta-1} \log ^{[2]} r\right] \cdot \frac{(A-\varepsilon)\left(\log ^{[2]} r\right)^{\alpha}}{\log r}, \\
& \frac{\log ^{[l]} T_{h}^{-1} T_{f \circ g}(r)}{\log r} \geqslant(\log r)^{(B-\varepsilon)(A-\varepsilon)^{\beta}\left(\log ^{[2]} r\right)^{\alpha \beta-1}} \cdot \frac{(A-\varepsilon)\left(\log ^{[2]} r\right)^{\alpha}}{\log r}, \\
& \limsup _{r \rightarrow \infty} \frac{\log ^{[l]} T_{h}^{-1} T_{f \circ g}(r)}{\log r} \geqslant \liminf _{r \rightarrow \infty}(\log r)^{(B-\varepsilon)(A-\varepsilon)^{\beta}\left(\log ^{[2]} r\right)^{\alpha \beta-1}} \cdot \frac{(A-\varepsilon)\left(\log ^{[2]} r\right)^{\alpha}}{\log r} .
\end{aligned}
$$

Since $\varepsilon>0$ is arbitrary and $\alpha>1, \alpha \beta>1$, it completes the proof.

Theorem 3.3. Let $f$ be a meromorphic function and $g, h$ be any two entire functions such that $0<\rho_{g} \leqslant \infty$ and $\lambda_{h}^{[l]}(f)>0$, where $l$ is any positive integer. Then

$$
\rho_{h}^{[l]}(f \circ g)=\infty .
$$

Proof. Suppose $0<\mu<\rho_{g} \leqslant \infty$.

As $T_{h}^{-1}(r)$ is an increasing function of $r$, we get from Lemma 2.1 for a sequence of values of $r$ tending to infinity that

$$
\begin{aligned}
& \log ^{[l]} T_{h}^{-1} T_{f \circ g}(r) \geqslant \log ^{[l]} T_{h}^{-1} T_{f}\left(\exp \left(r^{\mu}\right)\right), \\
& \log ^{[l]} T_{h}^{-1} T_{f \circ g}(r) \geqslant\left(\lambda_{h}^{[l]}(f)-\varepsilon\right) r^{\mu}
\end{aligned}
$$




$$
\begin{aligned}
& \frac{\log ^{[l]} T_{h}^{-1} T_{f \circ g}(r)}{\log r} \geqslant \frac{\left(\lambda_{h}^{[l]}(f)-\varepsilon\right) r^{\mu}}{\log r}, \\
& \limsup _{r \rightarrow \infty} \frac{\log ^{[l]} T_{h}^{-1} T_{f \circ g}(r)}{\log r} \geqslant \liminf _{r \rightarrow \infty} \frac{\left(\lambda_{h}^{[l]}(f)-\varepsilon\right) r^{\mu}}{\log r}, \\
& \rho_{h}^{[l]}(f \circ g)=\infty .
\end{aligned}
$$

Thus, the theorem follows.

Theorem 3.4. Let $f$ be a meromorphic function and $g, h$ be any two entire functions such that $0<\rho_{g} \leqslant \infty$ and $\lambda_{h}^{[l]}(f)>0$, where $l$ is any positive integer. Then

$$
\limsup _{r \rightarrow \infty} \frac{\log ^{[l]} T_{h}^{-1} T_{f \circ g}(r)}{\log ^{[l]} T_{h}^{-1} T_{f}(r)}=\infty .
$$

Proof. In view of Theorem 3.3, we obtain that

$$
\begin{aligned}
& \limsup _{r \rightarrow \infty} \frac{\log ^{[l]} T_{h}^{-1} T_{f \circ g}(r)}{\log ^{[l]} T_{h}^{-1} T_{f}(r)} \geqslant \limsup _{r \rightarrow \infty} \frac{\log ^{[l]} T_{h}^{-1} T_{f \circ g}(r)}{\log r} \liminf _{r \rightarrow \infty} \frac{\log r}{\log ^{[l]} T_{h}^{-1} T_{f}(r)}, \\
& \underset{r \rightarrow \infty}{\limsup } \frac{\log ^{[l]} T_{h}^{-1} T_{f \circ g}(r)}{\log ^{[l]} T_{h}^{-1} T_{f}(r)} \geqslant \rho_{h}^{[l]}(f \circ g) \cdot \frac{1}{\rho_{h}^{[l]}(f)}, \\
& \underset{r \rightarrow \infty}{\limsup } \frac{\log ^{[l]} T_{h}^{-1} T_{f \circ g}(r)}{\log ^{[l]} T_{h}^{-1} T_{f}(r)}=\infty .
\end{aligned}
$$

Thus, the theorem follows.

Theorem 3.5. Let $f$ be a meromorphic function and $h$ be an entire function such that $0<\lambda_{h}^{[l]}(f) \leqslant \rho_{h}^{[l]}(f)<\infty$. Also let $g$ be an entire function with a nonzero order. Then for every positive constant $A$ and every real number $\alpha$

$$
\limsup _{r \rightarrow \infty} \frac{\log ^{[l]} T_{h}^{-1} T_{f \circ g}(r)}{\left\{\log ^{[l]} T_{h}^{-1} T_{f}\left(r^{A}\right)\right\}^{1+\alpha}}=\infty,
$$

where $l$ is any positive integer.

Proof. If $\alpha$ be such that $1+\alpha \leqslant 0$ then the theorem is trivial. So we suppose that $1+\alpha>0$. Since $T_{h}^{-1}(r)$ is an increasing function of $r$, we get from Lemma 2.1 for a sequence of values of $r$ tending to infinity that

$$
\begin{aligned}
& \log ^{[l]} T_{h}^{-1} T_{f \circ g}(r) \geqslant \log ^{[l]} T_{h}^{-1} T_{f}\left(\exp \left(r^{\mu}\right)\right) \\
& \log ^{[l]} T_{h}^{-1} T_{f \circ g}(r) \geqslant\left(\lambda_{h}^{[l]}(f)-\varepsilon\right) r^{\mu},
\end{aligned}
$$

where we choose $0<\mu<\rho_{g} \leqslant \infty$. Again from the definition of $\rho_{h}^{[l]}(f)$, it follows for all sufficiently large values of $r$ that

$$
\begin{gathered}
\log ^{[l]} T_{h}^{-1} T_{f}\left(r^{A}\right) \leqslant\left(\rho_{h}^{[l]}(f)+\varepsilon\right) A \log r \\
\left\{\log ^{[l]} T_{h}^{-1} T_{f}\left(r^{A}\right)\right\}^{1+\alpha}
\end{gathered}
$$


Now from (3.5) and (3.6), it follows for a sequence of values of $r$ tending to infinity that

$$
\frac{\log ^{[l]} T_{h}^{-1} T_{f \circ g}(r)}{\left\{\log ^{[l]} T_{h}^{-1} T_{f}\left(r^{A}\right)\right\}^{1+\alpha}} \geqslant \frac{\left(\lambda_{h}^{[l]}(f)-\varepsilon\right) r^{\mu}}{\left(\rho_{h}^{[l]}(f)+\varepsilon\right)^{1+\alpha} A^{1+\alpha}(\log r)^{1+\alpha}} .
$$

Since $\frac{r^{\mu}}{(\log r)^{1+\alpha}} \rightarrow \infty$ as $r \rightarrow \infty$, the theorem follows from above.

Theorem 3.6. Let $f$ be a meromorphic function and $g$ be an entire function with a non-zero order. Also let $h$ and $k$ be any two entire function such that $0<\lambda_{h}^{[l]}(f)$ and $\rho_{k}(g)<\infty$, where $l$ is any positive integer. Then for every positive constant $A$ and every real number $\alpha$,

$$
\limsup _{r \rightarrow \infty} \frac{\log ^{[k]} T_{h}^{-1} T_{f \circ g}(r)}{\left\{\log T_{k}^{-1} T_{g}\left(r^{A}\right)\right\}^{1+\alpha}}=\infty .
$$

We omit the proof of Theorem 3.6 since it follows the same line as for Theorem 3.5.

Theorem 3.7. Let $f$ be a meromorphic function and $g$, $h$ be any two entire functions such that $\rho_{h}^{[l]}(f)<\infty$ and $\lambda_{h}^{[l]}(f \circ g)=\infty$. Then for every $\mu(>0)$,

$$
\lim _{r \rightarrow \infty} \frac{\log ^{[l]} T_{h}^{-1} T_{f \circ g}(r)}{\log ^{[l]} T_{h}^{-1} T_{f}\left(r^{\mu}\right)}=\infty
$$

where $l$ is any positive integer.

Proof. We argue by contradiction and assume that there exist a constant $\beta$ such that for a sequence of values of $r$ tending to infinity,

$$
\log ^{[l]} T_{h}^{-1} T_{f \circ g}(r) \leqslant \beta \cdot \log ^{[l]} T_{h}^{-1} T_{f}\left(r^{\mu}\right) .
$$

Again from the definition of $\rho_{h}^{[l]}(f)$, it follows for all sufficiently large values of $r$ that

$$
\log ^{[l]} T_{h}^{-1} T_{f}\left(r^{\mu}\right) \leqslant\left(\rho_{h}^{[l]}(f)+\varepsilon\right) \mu \log r .
$$

Now combining (3.7) and (3.8), we have for a sequence of values of $r$ tending to infinity that

$$
\begin{aligned}
\log ^{[l]} T_{h}^{-1} T_{f \circ g}(r) & \leqslant \beta \cdot\left(\rho_{h}^{[l]}(f)+\varepsilon\right) \mu \cdot \log r \\
\text { i.e., } \lambda_{h}^{[l]}(f \circ g) & \leqslant \beta \cdot \mu\left(\rho_{h}^{[l]}(f)+\varepsilon\right),
\end{aligned}
$$

which contradicts the condition $\lambda_{h}^{[l]}(f \circ g)=\infty$.

Hence, for all sufficiently large values of $r$ we get that

$$
\log ^{[l]} T_{h}^{-1} T_{f \circ g}(r) \geqslant \beta \cdot \log ^{[l]} T_{h}^{-1} T_{f}\left(r^{\mu}\right)
$$

that completes the proof.

Remark 3.1. Theorem 3.7 is also valid with "limit superior" instead of "limit" if $\lambda_{h}^{[l]}(f \circ g)=$ $\infty$ is replaced by $\rho_{h}^{[l]}(f \circ g)=\infty$ and the other conditions remain the same.

Corollary 1. Under the assumptions of Theorem 3.7 and Remark [3.1,

$$
\lim _{r \rightarrow \infty} \frac{T_{h}^{-1} T_{f \circ g}(r)}{T_{h}^{-1} T_{f}\left(r^{\mu}\right)}=\infty \quad \text { and } \quad \limsup _{r \rightarrow \infty} \frac{T_{h}^{-1} T_{f \circ g}(r)}{T_{h}^{-1} T_{f}\left(r^{\mu}\right)}=\infty
$$

respectively. 
Proof. By Theorem 3.7, we obtain for all sufficiently large values of $r$ and for $K>1$ that

$$
\begin{aligned}
\log ^{[l]} T_{h}^{-1} T_{f \circ g}(r) & \geqslant K \log ^{[l]} T_{h}^{-1} T_{f}\left(r^{\mu}\right) \\
\text { i.e., } \log ^{[l-1]} T_{h}^{-1} T_{f \circ g}(r) & \geqslant\left\{\log ^{[l-1]} T_{h}^{-1} T_{f}\left(r^{\mu}\right)\right\}^{K},
\end{aligned}
$$

from which the first part of the corollary follows. Similarly, using Remark 3.1, we obtain the second part of the corollary.

In the same way one may state the following theorem and corollaries; their proofs follows the same line as for Remark 3.1, Theorem 3.7 and Corollary 1, respectively.

Theorem 3.8. If $f$ be a meromorphic function and $g, h$ be any two entire functions such that $\rho_{h}^{[l]}(g)<\infty$ and $\rho_{h}^{[l]}(f \circ g)=\infty$, then for every $\mu(>0)$,

$$
\limsup _{r \rightarrow \infty} \frac{\log ^{[l]} T_{h}^{-1} T_{f \circ g}(r)}{\log ^{[l]} T_{h}^{-1} T_{g}\left(r^{\mu}\right)}=\infty
$$

where $l$ is any positive integer.

Corollary 2. Theorem 3.7 is also valid with "limit" instead of "limit superior" if $\rho_{h}^{[l]}(f \circ g)=$ $\infty$ is replaced by $\lambda_{h}^{[l]}(f \circ g)=\infty$ and the other conditions remain the same.

Corollary 3. Under the assumptions of Theorem 3.7 and Corollary 2 ,

$$
\limsup _{r \rightarrow \infty} \frac{T_{h}^{-1} T_{f \circ g}(r)}{T_{h}^{-1} T_{g}\left(r^{\mu}\right)}=\infty \quad \text { and } \quad \lim _{r \rightarrow \infty} \frac{T_{h}^{-1} T_{f \circ g}(r)}{T_{h}^{-1} T_{g}\left(r^{\mu}\right)}=\infty
$$

respectively.

\section{REFERENCES}

1. L. Bernal. Crecimiento relativo de funciones enteras. Contribución on al estudio de lasfunciones enteras conindice exponencial finito // PhD thesis, University of Seville, (1984).

2. L. Bernal. Orden relative de crecimiento de funciones enteras // Collect. Math. 39:3, 209-229 (1988).

3. W. Bergweiler. On the growth rate of composite meromorphic functions // Complex Variables, Theory Appl. 14:1-4, 187-196 (1990).

4. D. Banerjee and S. Jana. Meromorphic functions of relative order $(p, q)$ // Soochow J. Math. 33:3, 343-357 (2007).

5. S. K. Datta and T. Biswas. Growth of entire functions based on relative order // Int. J. Pure Appl. Math. 51:1, 49-58 (2009).

6. S. K. Datta and T. Biswas. Relative order of composite entire functions and some related growth properties // Bull. Cal. Math. Soc. 102:3, 259-266 (2010).

7. S. K. Datta, T. Biswas and D. C. Pramanik. On relative order and maximum term-related comparative growth rates of entire functions // J. Tripura Math. Soc. 14, 60-68 (2012).

8. S. K. Datta, T. Biswas and R. Biswas. On relative order based growth estimates of entire functions // Int. J. Math. Sci. \& Engg. Appls. 7:II, 59-67 (2013).

9. S. K. Datta, T. Biswas and R. Biswas. Comparative growth properties of composite entire functions in the light of their relative order // The Mathematics Student. 82:1-4, 209-216 (2013).

10. S. K. Datta, T. Biswas and C. Biswas. Growth analysis of composite entire and meromorphic functions in the light of their relative orders // Int. Scholarly Res. Notices. 2014, id 538327 (2014).

11. S. K. Datta, T. Biswas and C. Biswas. Measure of growth ratios of composite entire and meromorphic functions with a focus on relative order // Int. J. Math. Sci. \& Engg. Appls. 8:IV, 207-218 (2014). 
12. W.K. Hayman. Meromorphic Functions, The Clarendon Press, Oxford (1964).

13. B. K. Lahiri and D. Banerjee. Relative order of entire and meromorphic functions // Proc. Nat. Acad. Sci. India Ser. A. 69(A):3, 339-354 (1999).

14. D. Sato. On the rate of growth of entire functions of fast growth // Bull. Amer. Math. Soc. 69:3, 411-414 (1963).

15. G. Valiron. Lectures on the general theory of integral functions. Chelsea Publishing Company, New York (1949).

Sanjib Kumar Datta,

Department of Mathematics, University of Kalyani,

P.O.-Kalyani, Dist-Nadia, PIN- 741235, West Bengal, India.

E-mail: sanjib_kr_datta@yahoo.co.in

Tanmay Biswas,

Rajbari, Rabindrapalli, R. N. Tagore Road,

P.O.-Krishnagar, Dist-Nadia, PIN- 741101, West Bengal, India.

E-mail: tanmaybiswas_math@rediffmail.com

Pranab Das,

Department of Mathematics, University of Kalyani,

P.O.-Kalyani, Dist-Nadia, PIN- 741235, West Bengal, India.

E-mail: pranabdas90@gmail.com 